\title{
Ambientes colaborativos para a produção e o compartilhamento de atividades de aprendizagem: análise dos ambientes WebCollage, LDshake e ILDE
}

Patrícia Scherer Bassani, Universidade Feevale, patriciab@feevale.br

Carla Reis, Universidade Feevale, carla.cr.reis@gmail.com

Pedro Henrique Petry de Faria, Universidade Feevale, phpf_98@outlook.com

Resumo: Learning Design (LD) ou Design da Aprendizagem é uma área de pesquisa que tem por objetivo tornar mais explícito o processo de desenvolvimento de atividades de aprendizagem com o uso de tecnologias digitais, fomentando o uso de estratégias e de ferramentas para o registro e o compartilhamento dessas atividades. Este estudo, de abordagem qualitativa, teve como objetivo analisar ferramentas para representar $e$ compartilhar atividades de aprendizagem. A pesquisa foi organizada em duas etapas: a) revisão de literatura, para identificar ambientes citados em artigos científicos publicados no periódico Computers \& Education entre 2010-2019; b) a análise desses ambientes por meio da observação on-line. Resultados mostram que WebCollage e LDShake foram os ambientes mais citados. Entretanto, eles foram integrados a uma plataforma completa chamada Integrated Learning Design Environment (ILDE).

Palavras-chave: tecnologia educacional; design da aprendizagem; artefato de mediação

\section{Collaborative environments for production and sharing of learning activities: analysis of WebCollage, LDshake and ILDE environments}

Abstract: Learning Design (LD) is a research area which aims to make the process of developing learning activities using digital technologies more explicit, encouraging the use of strategies and tools for documentation and sharing. This study, based on a qualitative approach, aims to analyse tools for representing and sharing learning activities. The research was organized into two stages: a) literature review to identify learning design environments cited in papers published in the journal Computers \& Education during 2010-2019; b) the analysis of these environments through online observation. Results show that Webcollage and LDshake were the most cited online environments for producing and sharing learning designs. However, they have been integrated into a complete platform called Integrated Learning Design Environment (ILDE).

Keywords: educational technology; learning design; mediation artifact

\section{Introdução}

O Learning Design (LD) ou Design da Aprendizagem é uma área de pesquisa que tem por objetivo tornar mais explícito o processo de desenvolvimento de atividades de aprendizagem com o uso de tecnologias digitais.

A área de LD fomenta a pesquisa a partir de três conceitos-chave: orientação (guidance), representação (representation) e compartilhamento (sharing). Pesquisas na perspectiva da orientação abordam as diferentes formas/maneiras nas quais os educadores podem ser auxiliados no processo de aprender e de utilizar novos métodos envolvendo tecnologias digitais. Pesquisas na perspectiva da representação focam no desenvolvimento de propostas (ferramentas) para representar as diferentes práticas relacionadas às atividades de aprendizagem. Por fim, pesquisas com foco no conceito de compartilhamento concentram-se na importância de compartilhar boas ideias com 
outros educadores, a fim de impulsionar o uso das tecnologias digitais no contexto educativo (THE LARNACA DECLARATION ON LEARNING DESIGN, 2013).

O LD oportuniza formas de representar as atividades de aprendizagem permitindo que elas sejam compartilhadas (CONOLE, 2008). Pode-se representar diferentes níveis de granularidade, envolvendo desde uma atividade individual até a concepção de um curso (CONOLE, 2013).

As sequências de atividades de aprendizagem podem ser codificadas em diferentes formas de representação. Para Conole (2008), estas formas de representação são conhecidas como artefatos de mediação, em função do seu papel de mediar o design das atividades. Conforme Conole (2008), o que deve ser documentado é a essência de uma atividade de aprendizagem, a fim de possibilitar que ela seja reutilizada no desenvolvimento de uma nova atividade, considerando-se diferentes contextos.

A mesma atividade de aprendizagem pode ser representada de diferentes formas: estudo de caso (texto), linguagem formal (especificação), representação visual, vocabulários (taxonomias, ontologias, folksonomias). Os artefatos de mediação diferenciam-se pela forma de representação (textual, visual, etc.), mas também isto implica em diferenças no grau de abstração e no detalhamento das informações disponíveis. Dessa forma, diferentes artefatos de mediação destacam diferentes aspectos de uma atividade de aprendizagem (BASSANI et al, 2016a).

A documentação das atividades de aprendizagem, por meio de artefatos de mediação, permite a construção de um banco de dados de práticas educativas. Assim, essas práticas podem ser compartilhadas e acessadas por diferentes professores, que podem reutilizá-las, modificá-las e validá-las em diferentes contextos (BASSANI et al, 2016a).

Assim, é importante destacar que LD abrange tanto o processo de projetar uma experiência de aprendizagem quanto o produto, ou seja, o artefato de mediação que representa uma atividade de aprendizagem (CONOLE, 2013).

Laurillard (2012), aponta que o processo de ensino é normalmente uma atividade muito pessoal e uma sequência de atividades existe apenas na cabeça do professor que a implementa. A pesquisadora destaca que a comunidade de professores deve contribuir para a construção de conhecimento sobre práticas com tecnologias, e isso deve ser feito de forma colaborativa. Além disso, os professores devem exercitar o processo de design das atividades de aprendizagem como parte de sua prática profissional.

Nessa perspectiva, entendemos que a documentação e o compartilhamento das atividades de aprendizagem desenvolvidas no contexto da educação formal podem impulsionar o uso das tecnologias digitais na sala de aula, uma vez que os professores de diferentes áreas e de várias escolas/instituições podem acessar e usar diferentes propostas para ensinar e aprender com o uso de tecnologias digitais, superando um modelo fortemente relacionado apenas com atividades de pesquisa na internet, conforme documentado nas pesquisa nacionais (CGI.br, 2017). Além disso, possibilita a criação de um conjunto de práticas que podem ser compartilhadas, reutilizadas e remixadas, oportunizando a colaboração em rede entre os professores.

As pesquisas na área de LD relacionam-se aos estudos sobre o uso de recursos educacionais abertos (REA). REA "são materiais de ensino, aprendizado e pesquisa, fixados em qualquer suporte ou mídia, que estejam sob domínio público ou licenciados de maneira aberta, permitindo que sejam utilizados ou adaptados por terceiros" (REA, 2019, on-line). Conforme Rossini e Gonzales (2012, p. 39), "a filosofia dos recursos educacionais abertos coloca os materiais educacionais na posição de bens comuns e públicos, voltados para o benefício de todos". Dessa forma, o foco das iniciativas REA envolve a disponibilização e o compartilhamento destas unidades, "que podem ser 
remixadas, traduzidas e adaptadas para finalidades educacionais, como as peças de um grande quebra-cabeças, transformando a forma como a educação é pensada e desenvolvida" (ROSSINI; GONZALES, 2012, p. 39). A cultura do remix pode ser definida como a atividade que consiste na modificação e na criação livre de novos artefatos, possibilitada pelas características das tecnologias digitais, suportada pela prática de cortar/copiar e colar, e pela dinâmica da sociedade contemporânea (LEMOS, 2006).

Existem diferentes ambientes colaborativos que podem ser utilizados para o registro e o compartilhamento de atividades de aprendizagem (CONOLE, 2013; BASSANI, 2014). Este estudo tem por objetivo investigar os ambientes de learnig design mais utilizados a partir de uma revisão sistemática de literatura e analisar as características destes ambientes a partir de duas perspectivas: a) modelo de artefato de mediação utilizado; b) modelo de colaboração utilizado para o compartilhamento em rede.

A pesquisa parte do seguinte questionamento: Quais ambientes para a produção e o compartilhamento de atividades de aprendizagem são mais citados nas pesquisas na área e quais as características destes ambientes? Esta pesquisa se articula ao projeto "Práticas educativas em/na rede: autoria e colaboração no desenvolvimento e atividades de aprendizagem com tecnologias digitais " ", desenvolvido na Universidade Feevale.

O artigo está assim organizado: na seção 2 apresentamos o delineamento metodológico do estudo; na seção 3, os resuldados, e na seção 4, a discussão. Por fim, as considerações finais, seguidas das refêrências deste estudo.

\section{Metodologia}

Este estudo se articula ao projeto de pesquisa "Práticas educativas em/na rede: autoria e colaboração no desenvolvimento e atividades de aprendizagem com tecnologias digitais", que tem por objetivo propor um ambiente para o registro e o compartilhamento de atividades de aprendizagem com tecnologias.

A pesquisa aqui apresentada foi desenvolvida em duas etapas. A primeira etapa envolveu uma revisão sistemática de literatura, para identificar os ambientes de learnig design citados em artigos científicos publicados no período 2010-2019. A segunda etapa envolveu a análise desses ambientes por meio de observação on-line. Cada uma das estas está descrita a seguir.

\subsection{Revisão de literatura}

A pesquisa foi inspirada na proposta de revisão sistemática de literatura desenvolvida por Kitchenham e Charters (2007). O processo de pesquisa iniciou com a definição da questão de pesquisa (RQ - research question): Quais as ferramentas e/ou ambientes utilizados para o registro e o compartilhamento de atividades de aprendizagem a partir de estudos na área de Learning Design?

A coleta de dados foi realizada na base de artigos publicados no periódico Computers \& Education ${ }^{2}$ (ISSN 0360-1315). Esse periódico foi selecionado pelo seu foco interdisciplinar na temática de tecnologia educacional. Além disso, o periódico é classificado como A nas áreas de Educação (A2), Ciência da Computação (A1) e Interdisciplinar (A2) no Qualis da Capes, e seu fator de impacto é 2018:5.627.

A palavra-chave utilizada no sistema foi "learning design" e a busca retornou 155 artigos, considerando-se os anos de 2010 a 2019.

\footnotetext{
${ }^{1}$ O projeto encontra-se aprovado no Comitê de Ética protocolo número: CAAE: 05576818.8.0000.5348. ${ }^{2}$ https://www.journals.elsevier.com/computers-and-education
} 
O conjunto de artigos foi exportado para o ambiente on-line RAYYAN QCRI (https://rayyan.qcri.org/), um software que tem por objetivo facilitar o processo de revisão sistemática. Uma segunda palavra-chave ("tool") foi aplicada no conjunto de artigos selecionados, a fim de identificar apenas artigos que tratassem de ferramentas. A ferramenta Rayyan faz a seleção de palavras-chave com base no resumo. Esta busca retornou 22 artigos.

A seguir, realizamos a leitura dos resumos destes 22 artigos. Do total de artigos analisados, apenas 2 focaram no uso de ferramentas de learning design, conforme mostra o Quadro 1.

Quadro 1. Análise de artigos sobre Learning Design

\begin{tabular}{|l|c|l|}
\hline \multicolumn{1}{|c|}{ Tema do artigo } & $\begin{array}{c}\text { Quantidade } \\
\text { de artigos }\end{array}$ & \multicolumn{1}{|c|}{ Detalhamento } \\
\hline $\begin{array}{c}\text { Learning design: } \\
\text { área de abrangência }\end{array}$ & 16 & $\begin{array}{l}\text { Pesquisas diversas sobre cursos e } \\
\text { projetos a presenciais e a distância }\end{array}$ \\
\hline $\begin{array}{l}\text { Learning design: } \\
\text { planejamento }\end{array}$ & 4 & $\begin{array}{l}\text { Cenários de aprendizagem; planos } \\
\text { pedagógicos }\end{array}$ \\
\hline $\begin{array}{l}\text { Learning Design: } \\
\text { ferramentas e/ou ambientes }\end{array}$ & 2 & $\begin{array}{l}\text { - Web Collage } \\
\text { - LdShake }\end{array}$ \\
\hline
\end{tabular}

A partir do estudo realizado, identificamos dois ambientes utilizados para o registro e o compartilhamento de atividades de aprendizagem: Web Collage e LdShake.

Um segundo momento envolveu a análise desses ambientes, conforme mostra a próxima seção.

\subsection{Análise dos ambientes}

A segunda etapa envolveu a observação on-line (HEWSON; LAURENT, 2012; SANTOS; COSTA, 2015) dos ambientes LdShake e Web Collage, a fim de identificar os modelos de registro e de compartilhamento.

A observação on-line dos ambientes levou em conta as seguintes características: a) idioma; b) modelo de registro de uma atividade de aprendizagem; c) proposta de compartilhamento.

Para analisar o modelo de registro de uma atividade foram utilizados os seguintes critérios (BASSANI et al, 2016a):

a) modelo de artefato de mediação utilizado, com base nos estudos de LD; (texto, visual, slides, etc);

b) modelo de licenciamento, com base nas licenças creative commons ${ }^{3}(\mathrm{CC})$;

c) autoria, a fim de verificar a origem dos materiais publicados;

d) normas de publicação, a fim de verificar se os documentos passam por alguma avaliação/revisão antes de serem publicados no repositório - exige aprovação prévia?;

e) link para recursos digitais externos, na perspectiva de indicação e/ou articulação com recursos educacionais abertos ou outras fontes complementares de conteúdo na web;

f) possibilidade de remixar para (re)utilizar, ou seja, usar uma sequência de atividades como base e modificá-la, (re)publicando-a no repositório.

\footnotetext{
${ }^{3}$ O Creative Commons é uma organização não-governamental que tem como foco a elaboração e manutenção de licenças livres para fomentar a cultura do compartilhamento. Para saber mais: http://educacaoaberta.org/cadernorea/licen\%C3\%A7as
} 
O modelo de compartilhamento foi analisado com base nos critérios propostos por Bassani et al (2016b). Esses critérios foram elaborados a partir de estudos sobre modelos de colaboração em ambientes colaborativos. As características de colaboração envolvem a comunicação, a coordenação, a cooperação e a conexão. A comunicação consiste basicamente na troca de mensagens entre os indivíduos. A coordenação pode ocorrer no nível de atividades (gerenciamento de atividades colaborativas) e/ou no nível de objetos (produção de documentos/artefatos colaborativos). A cooperação refere-se ao trabalho em conjunto em um ambiente compartilhado. A conexão aparece como forma de permitir que os usuários conectem-se a outros usuários e a conteúdos, na perspectiva de formação de redes sociais.

\section{Resultados}

Apresentamos aqui os resultados da observação on-line dos ambientes.

\subsection{Web Collage (https://webcollage.gsic.uva.es/)}

$\mathrm{O}$ ambiente Web Collage permite a documentação de atividades de aprendizagem por meio de um modelo visual (Figura 1). O ambiente está disponível em Inglês e Espanhol. Para cada registro de atividade (chamado de design), podem ser indicados os prerrequisitos e os objetivos da aprendizagem.

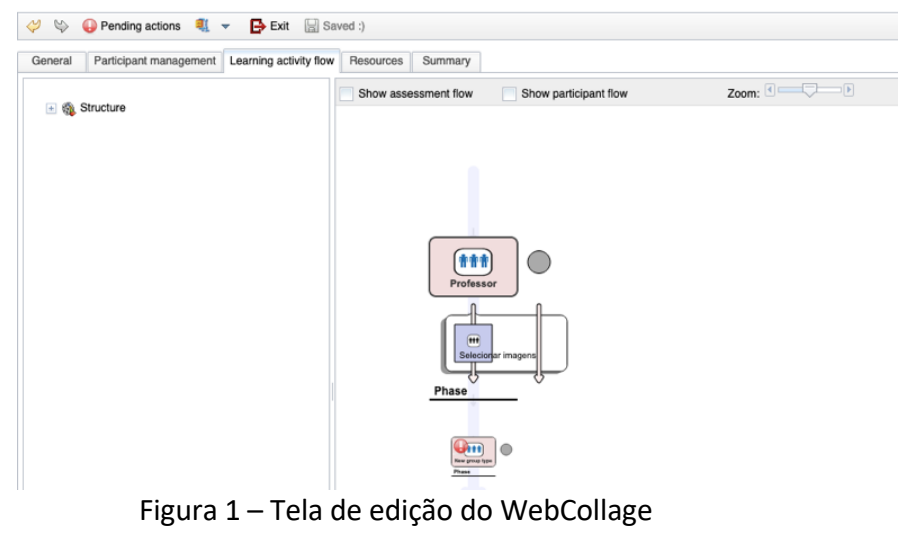

Em relação ao modelo de registro da atividade, não foi possível identificar nenhum dos critérios analisados. Em relação ao modelo de compartilhamento, verificamos que não existe a possibilidade de compartilhar com os outros usuários; apenas permite exportar em forma de script ou .pdf. Verificamos que o WebCollage foi incorporado ao ambiente Integrated Learning Design Environment (ILDE).

3.2 LdShake (http://ldshake.upf.edu/demo/)

O LDShake é um ambiente web desenvolvido pelo grupo de pesquisa GTI (Interactive Technologies Group), da Universidade Pompeu Fabra, de Barcelona (Espanha). É um ambiente onde os professores podem co-editar e compartilhar atividades de aprendizagem ("A site where teachers (LdShakers) co-edit and share Learning design Solutions (LdS)"). A Figura 2 mostra a tela de edição do ambiente. 


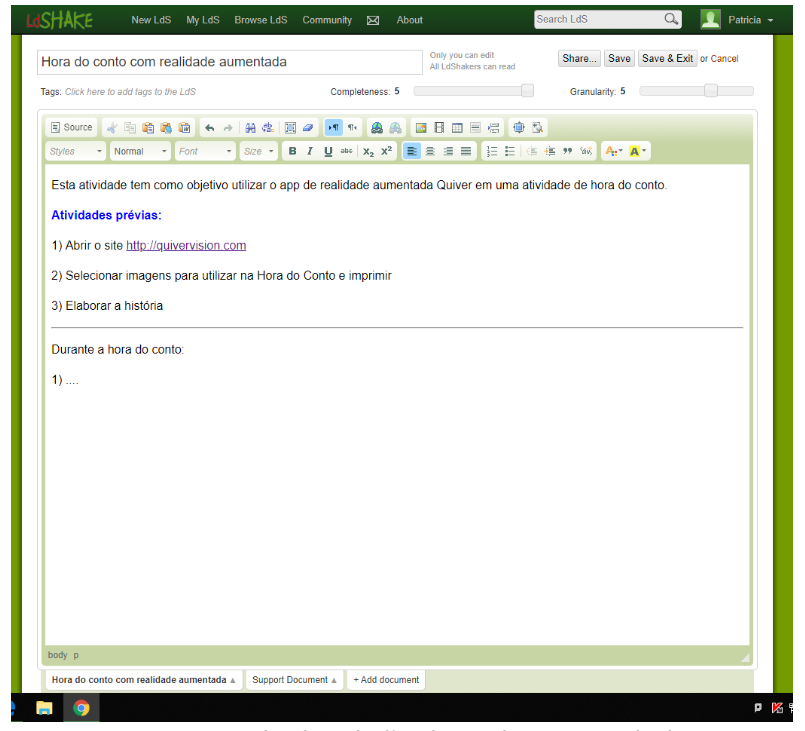

Figura 2. Tela de edição do ambiente LDShake

Em relação ao modelo de registro das atividades, verificamos que o artefato de mediação tem formato livre (texto) e ainda existe a possibilidade de importar um modelo visual do WebCollage. Permite links para recursos externos e também a inserção de imagens e vídeos. Cada learning design pode ser classificado em relação a sua granularidade (atividade, módulo, curso e outras). A autoria é livre e não há revisão por parte de mediadores do ambiente. Além disso, cada autor/a tem a possibilidade de indicar o tipo de licença do recurso produzido, com base nas licenças Creative Commons (CC).

Todo artefato (learning design) produzido no LDShake fica disponível aos demais usuários para remix, ou seja, o sujeito pode fazer uma cópia do artefato e inserir suas adaptações/modificações, produzindo uma nova proposta.

Em relação ao modelo de compartilhamento, destacamos o seguinte: no ambiente;

a) comunicação: permite o envio de mensagens para qualquer usuário cadastrado

b) cooperação: permite a edição compartilhada de artefatos (vários autores podem editar de forma não simultânea o mesmo learning design);

c) coordenação: não permite edição simultânea de artefatos - se um autor está editando, o outro precisa aguardar a liberação;

d) conexão: permite a criação de grupos de usuários. Outra questão relevante é que os documentos produzidos podem ser publicados na web. Ao clicar em publish, o sistema gera um link público para acesso direto ao learning design. Isso é interessante, pois o professor pode compartilhar sua proposta de atividade em outros ambientes, para inspirar outros professores, ou ainda disponibilizar acesso ao conteúdo para os seus alunos.

Verificamos que este ambiente, assim como o WebCollage, também foi incorporado ao ambiente Integrated Learning Design Environment (ILDE). Assim, decidimos analisar este ambiente também.

\subsection{Integrated Learning Design Environment (ILDE) (http://ilde.upf.edu)}

$\mathrm{O}$ ambiente ILDE, desenvolvido pelo Grupo de Pesquisa TIDE (Interactive \& Distributed Technologies for Education) da Universidade Pompeu Fabra, de Barcelona (Espanha), amplia a proposta do LDShake e caracteriza-se como "the all-in-one learning design community platform".

O ILDE incorpora diferentes ferramentas de learning design disponíveis na web, para cobrir todo o ciclo do learning design, incluindo ferramentas para: 
a) conceitualização (ferramentas para a concepção e para o planejamento de cursos/atividades);

b) autoria (ferramentas para autoria de projetos, incluindo aqui a documentação de atividades de aprendizagem): o learning design pode ser produzido usando o modelo de fluxo do WebCollage, o modelo de texto livre do LDShake, ou ainda permite acesso as ferramentas de autoria do GoogleDrive (planilhas, apresentações, texto). A Figura 3 apresenta as possibilidade de autoria de um learning design no ambiente ILDE; Moodle).

c) implementação (articulação direta com o ambiente virtual de aprendizagem

O ambiente disponibiliza a tradução para sete línguas, sendo elas: inglês, espanhol, alemão, italiano, grego, hebraico e catalã.

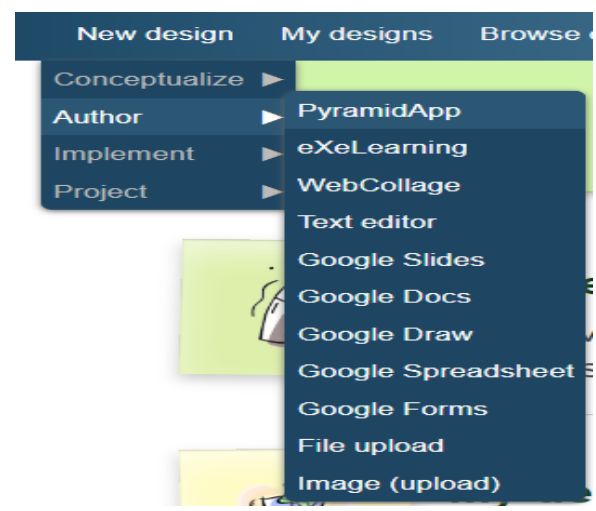

Figura 3. Diferentes possibilidade para o registro de um learning design no ambiente ILDE

Em relação ao modelo de registro, o ILDE incorpora diferentes possibilidades, mas segue os mesmos critérios do LDShake em relação ao uso de recursos externos, licenciamento e remix.

O ILDE também segue o mesmo modelo de compartilhamento do ambiente LDShake, fomentando o acesso aos documentos por toda a comunidade de usuários. Os documentos produzidos no ambiente podem ser localizados por meio de palavras-chave definidas pelos autores. Infelizmente não há uma busca direta no sistema para localizar artefatos produzidos em um determinado país ou para uma disciplina/conteúdo específico. analisados.

O Quadro 2 apresenta um resumo do modelo de registro dos três ambientes

Quadro 2. Quadro-resumo do modelo de registro dos ambientes WebCollage, LDSHake e IDLE

\begin{tabular}{|c|c|c|c|c|c|c|}
\hline Ferramenta & Artefato & Licenciamento & Autoria & Normas & Link & Remix \\
\hline LdShake & $\begin{array}{c}\text { Texto livre- } \\
\text { permite importar } \\
\text { modelo do } \\
\text { WebCollage }\end{array}$ & $\begin{array}{c}\text { Definido pelo } \\
\text { autor }\end{array}$ & $\begin{array}{c}\text { Qualquer } \\
\text { usuário pode } \\
\text { cadastrar } \\
\text { ILDE }\end{array}$ & $\begin{array}{c}\text { Não existe } \\
\text { nenhuma } \\
\text { avaliação/ } \\
\text { revisão de } \\
\text { documento }\end{array}$ & $\begin{array}{c}\text { Sim, permite } \\
\text { link e } \\
\text { incorporação } \\
\text { de recursos } \\
\text { externos }\end{array}$ & $\begin{array}{c}\text { Sim, } \\
\text { independente } \\
\text { da licença }\end{array}$ \\
& $\begin{array}{c}\text { Texto livre- } \\
\text { permite importar } \\
\text { modelos de } \\
\text { outras } \\
\text { plataformas } \\
\text { GoogleDrive } \\
\text { outras) }\end{array}$ & $\begin{array}{c}\text { Definido pelo } \\
\text { autor }\end{array}$ & $\begin{array}{c}\text { Qualquer } \\
\text { usuário pode } \\
\text { cadastrar }\end{array}$ & $\begin{array}{c}\text { Não existe } \\
\text { nenhuma } \\
\text { avaliação/ } \\
\text { revisão de } \\
\text { documento }\end{array}$ & $\begin{array}{c}\text { Sim, permite } \\
\text { link e } \\
\text { incorporação } \\
\text { de recursos } \\
\text { externos }\end{array}$ & $\begin{array}{c}\text { Sim, } \\
\text { datependente licença }\end{array}$ \\
\hline
\end{tabular}




\begin{tabular}{|c|c|c|c|c|c|c|}
\hline Ferramenta & Artefato & Licenciamento & Autoria & Normas & Link & Remix \\
\hline $\begin{array}{c}\text { Web } \\
\text { Collage }\end{array}$ & $\begin{array}{c}\text { Fluxo, a partir } \\
\text { de modelos pré- } \\
\text { definidos }\end{array}$ & $\begin{array}{c}\text { Não } \\
\text { identificado }\end{array}$ & $\begin{array}{c}\text { Não } \\
\text { identificado }\end{array}$ & $\begin{array}{c}\text { Não } \\
\text { identificado }\end{array}$ & $\begin{array}{c}\text { Não } \\
\text { identificado }\end{array}$ & $\begin{array}{c}\text { Não } \\
\text { identificado }\end{array}$ \\
\hline
\end{tabular}

A próxima seção apresenta a discussão dos resultados à luz dos estudos sobre LD.

\section{Discussão}

Os ambientes foram analisados a partir de duas perspectivas: a) modelo de registro; b) modelo de compartilhamento. Os processos de registro e de compartilhamento são conceitos-chave no contexto dos estudos de LD.

$\mathrm{O}$ modelo de registro, aqui entendido como learning design ou artefato de mediação (CONOLE, 2013), varia conforme o ambiente analisado. Foram utilizados os critérios propostos pode Bassani et al. (2016a). O WebCollage usa um modelo visual (fluxo). O LDShake disponibiliza o formato de texto livre. Por outro lado, o ILDE permite diferentes modelos, seja fluxograma (incorporando o WebCollage), texto livre (incorporando o LDShake), ou ainda propostas que incluem o uso das ferramentas do GoogleDrive. Assim, verificamos que não há um padrão para o registro de atividades de aprendizagem, uma vez que cada autor/a pode escolher o modelo mais adequado para as suas necessidades. Essa proposta livre é interessante, uma vez que pode ser adequada a qualquer nível de granularidade (uma atividade, um conjunto de atividades, um módulo, um curso, etc).

Os ambiente LDShake e ILDE têm em comum a possibilidade de remix e também o registro de licenciamento com base no creative commons, questões relevantes que impactam na reutilização e no compartilhamento dos documentos.

A análise do modelo de compartilhamento teve por objetivo identificar o potencial dos ambientes na perspectiva da colaboração. O modelo de colaboração foi analisado a partir de quatro critérios propostos por Bassani et al. (2016b).

O ambiente WebCollage não apresenta características de colaboração. Por outro lado, os ambientes LDShake e ILDE permitem a formação de grupos, o compartilhamento e a reutilização dos artefatos de mediação produzidos de forma individual ou coletiva. Entretanto, a edição coletiva possui limitações, uma vez que não existe a possibilidade de edição simultânea de artefatos. Por outro lado, a possibilidade de compartilhamento público na web, por meio de um link de livre acesso ao artefato produzido, permite que a proposta de atividade desenvolvida de forma individual e/ou coletiva seja compartilhada em blogs, sites ou redes sociais. Isso permite que uma proposta alcance uma audiência maior.

Entendemos que o modelo de compartilhamento disponível nos ambientes LDShake e ILDE pode ajudar a divulgar as boas ideias de atividades produzidas e fomentar a autoria docente, conforme a proposta de Laurillard (2012).

\section{Considerações finais}

A pesquisa teve como ponto de partida o seguinte questionamento: Quais ambientes para a produção e o compartilhamento de atividades de aprendizagem são mais citados nas pesquisas na área e quais as características destes ambientes? Por meio do percurso de pesquisa trilhado identificamos que os ambientes WebCollage e LDShake podem ser utilizados de forma isolada, mas também foram integrados à plataforma ILDE.

A plataforma ILDE integra diferentes ferramentas de learning design produzidas por vários grupos de pesquisa e disponíveis na web e pode ser realmente considerada uma plataforma all-in-one. 
A plataforma ILDE tem potencial para centralizar a produção de learning designs. A próxima etapa de pesquisa consiste em validar este ambiente, a fim de verificar o efetivo potencial de uso e de engajamento dos professores na proposta.

\section{Referências}

BASSANI, Patricia. B. Scherer. Documentação de atividades de aprendizagem com o uso de tecnologias In: JAIE 2014 - 3a Jornada de Atualização em Informática na Educação. 1 ed.Assis/SP : Triunfal Gráfica e Editora, 2014a, p. 107-144.

BASSANI, Patricia. B. Scherer; LIMA, C. C. ; DALANHOL, D. R. Documentação e compartilhamento de atividades de aprendizagem: um estudo sobre repositórios de prática e artefatos de mediação. Revista e-Curriculum (PUCSP), v. 14, p. 1423-1453, 2016 a.

BASSANI, Patricia. B. Scherer; REIS, A. N.; DALANHOL, D. R. Análise da colaboração em ambientes digitais para compartilhamento de atividades de aprendizagem: uma perspectiva com base em Learning Design In: 27o Simpósio Brasileiro de Informática na Educação - SBIE, 2016, Uberlândia. Anais do XXVII Simpósio Brasileiro de Informática na Educação (SBIE 2016). Porto Alegre: Sociedade Brasileira de Computação - SBC, 2016 b.

COMITÊ GESTOR DA INTERNET NO BRASIL - CGI.br. Pesquisa Sobre o Uso da Internet por crianças e adolescentes no Brasil- TIC Kids Online Brasil 2016. São Paulo: CGI.br, 2017.

CONOLE, Grainne. Capturing practice: the role of mediating artefacts in learning design. In: LOCKYER, Lori et al. (Eds). Handbook of research on learning design and learning objects. Hersey, PA: IGI Global, 2008. p. 187 - 207.

CONOLE, Grainne. Designing for learning in an open world. UK: Springer, 2013.

HERNÁNDEZ-LEO, Davinia. ROMEO, Lauren. A. CARRALERO, Miguel. CHACÓN, Jonathan. CARRIÓ, Mar. MORENO, Pau. BLAT, Josep. LdShake: Learning design solutions sharing and co-edition. Revista Computer \& Education, espanha, v. 57, n.4, 2011 Disponivel em : https://doi.org/10.1016/j.compedu.2011.06.016

HEWSON, Claire; LAURENT, Dianna. Research design and tools for internet research. In: HUGHES, Jason. (Ed.). Sage internet research methods. Sage: London, 2012. p. 165-193.

ILDE. Disponível em < https://ilde.upf.edu/> Acesso em: 22 set. 2019

KITCHENHAM, B.; CHARTERS, S. Guidelines for performing systematic literature reviews in software engineering. Technical Report EBSE-2007-01. 2007.

LAURILLARD, Diana. Teaching as a design science. New York: Taylor \& Francis, 2012.

LDSHAKE. Disponível em < http://ldshake.upf.edu/demo/ > Acesso em: 22 set. 2019.

LEMOS, André. Ciber-cultura-remix. In: ARAUJO, Denize Correa. (Org.). Imagem (Ir) realidade: comunicação e cibermídia. Porto Alegre: Sulina, 2006.

REA - Recursos Educacionais Abertos. Disponível em: $<$ http://www.rea.net.br/site/conceito $>$. Acesso em: 22 set. 2019.

ROSSINI, Carolina; GONZALES, Cristiana. REA: o debate em política pública e as oportunidades para o mercado. In: SANTANA, Bianca et al. (Orgs.). Recursos 
Educacionais Abertos: práticas colaborativas políticas públicas, Salvador: Edufba; São Paulo: Casa da Cultura Digital, 2012, p. 35-71.

SANTOS, Vera Lúcia; COSTA, Cleide. A observação online como instrumento investigativo. Debates em Educação, vol. 7, n. 15, jul/dez 2015. Disponível em: < http://www.seer.ufal.br/index.php/debateseducacao/article/view/1339/1568>. Acesso em: 20 abr. 2016.

The Larnaca Declaration on Learning Design. Disponível em: https://larnacadeclaration.wordpress.com/. 2013.

VILLASCLARAS-FERNÁNDEZ, Elloy. HERNÁNDEZ-LEO Davinia. ASENSIOPÉREZ, Juan I. DIMITRIADIS, Yannis. Web Collage: An implementation of support for assessment design in CSCL macro-scripts. Revista Computer \& Education, espanha, v. 67, 2013 Disponivel em: https://doi.org/10.1016/j.compedu.2013.03.002

WEB COLLAGE. Disponível em: $<$ https://webcollage.gsic.uva.es/)> . Acesso em: 22 set. 2019. 